Career situation of first and presenting author Student for a master or a $\mathrm{PhD}$.

Introduction Endothelial cells (EC) are important contributors to inflammation via expression of inflammatory mediators, including chemokines and adhesion molecules. Production of these inflammatory mediators can be induced via canonical and NF- $\kappa \mathrm{B}$-inducing kinase (NIK)-dependent noncanonical NF$\kappa \mathrm{B}$ signalling. The ligands activating these pathways are well studied, but less is known about the cells producing ligands that can activate NF- $\mathrm{BB}$ signalling in EC.

Objectives To study the effects of soluble factors produced by activated memory $\mathrm{T}\left(\mathrm{T}_{\mathrm{m}}\right)$ cells on NF- $\kappa \mathrm{B}$ dependent inflammatory activation of EC.

Methods $\mathrm{CD}^{+} \mathrm{CD}_{45 \mathrm{RO}^{+}}$Memory $\mathrm{T}$ cells were isolated from healthy PBMC using MACS sorting and cultured in medium containing anti-CD3 and anti-CD28 for 72 hour, after which supernatant was harvest. Human umbilical cord EC (HUVEC) were stimulated with $50 \% \mathrm{~T}_{\mathrm{m}}$ supernatant $\left(\mathrm{T}_{\mathrm{m}}\right.$ sup). After 72 hour of $\mathrm{T}_{\mathrm{m}}$ sup stimulation HUVEC protein and RNA was harvest and $\mathrm{NF}-\mathrm{\kappa B}$ signalling and downstream expression of inflammatory mediators was analysed using qPCR and Western Blot. Culture supernatant was analysed by ELISA to detect presence of inflammatory mediators. To repress canonical NF- $\kappa \mathrm{B}$ signalling an inhibitor of IKK $\beta$ (iIKK $\beta$ ) was used and to repress NIK-dependent NF$\kappa \mathrm{B}$ signaling an inhibitor of NIK (iNIK) was used.

Results Stimulation with $\mathrm{T}_{\mathrm{m}}$ sup led to activation of both canonical NF- $\kappa \mathrm{B}$ signalling, indicated by increased levels of phosphorylated ( $\mathrm{p})-\mathrm{I} \kappa \mathrm{B} \alpha$, and noncanonical NF- $\mathrm{B}$ signalling, indicated by increased p100 to p52 processing. HUVEC stimulated with $T_{m}$ sup had increased mRNA levels of all tested inflammatory mediators compared to non-treated cells. Gene expression of chemokines (CXCL1, CXCL5, IL6, IL8 and GM-CSF) after $T_{m}$ sup stimulation was significantly reduced after treatment with iIKK $\beta$ and to a lesser, but still significant, extent after treatment with iNIK. Interestingly, treatment with iIKK $\beta$ also led to a reduction in mRNA levels of the adhesion molecules VCAM-1 and ICAM-1, while this effect was minimal after iNIK treatment. In addition, treatment with either IKK $\beta$ or iNIK led to a significant reduction in CXCL5 in the culture supernatant of HUVEC stimulated with $\mathrm{T}_{\mathrm{m}}$ sup.

Conclusions This study provides new insights into the cellular interactions leading to production of inflammatory mediators by EC. Our findings demonstrate that activated $T_{m}$ cells fac-

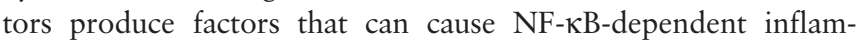
matory activation of EC. Targeting canonical NF- $\kappa B$ signaling

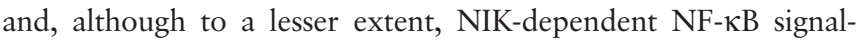
ing reduces inflammatory activation of the endothelium.

Disclosure of Interest None declared.

\section{P086 SYSTEMIC OVEREXPRESSION OF INTERLEUKIN-22 INDUCES EXPRESSION OF THE NEGATIVE IMMUNE- REGULATOR SOCS3 AND POTENTLY REDUCES COLLAGEN-INDUCED ARTHRITIS IN MICE}

J Aarts*, D Roeleveld, B Walgreen, M Helsen, E Vitters, F Van de Loo, P Van Lent, W Van den Berg, P Van der Kraan, M Koenders. Experimental Rheumatology, Radboud University Medical Center, Nijmegen, Netherlands

\subsection{6/annrheumdis-2018-EWRR2019.75}

Career situation of first and presenting author Student for a master or a $\mathrm{PhD}$.

Introduction High interleukin-22 (IL-22) levels are detected in serum and synovial fluid of rheumatoid arthritis (RA) patients and have been shown to positively correlate with disease activity markers and more erosive disease. The role of IL-22 in autoimmunity and inflammation appears to be greatly contradictory, being both pro- and anti-inflammatory. Especially the antiinflammatory properties of IL-22 are not well understood. We aimed to investigate the anti-inflammatory and immune-suppressive effect of IL-22 during experimental arthritis.

Methods Collagen-induced arthritis was induced in DBA1 mice by immunization and booster with bovine collagen type II (CII). After booster, but before arthritis onset, IL-22 was overexpressed either locally or systemically using an adenoviral construct (AdIL-22) or Luciferase as control (AdLuc). $1 \times$ $10^{7}$ plaque-forming units (PFU) of the adenoviruses were injected intra-articularly for local overexpression, or $3 \times$ $10^{8}$ PFU was injected intravenously for systemic overexpression in immunized mice, and mice were sacrificed 10 days later. Macroscopic scoring and histological analysis was performed, and mRNA expression and protein production of various pro- and anti-inflammatory mediators was determined in synovial tissue, spleen, and serum.

Results Local overexpression of IL-22 by injection of AdIL-22 in the knee joint of CII-immunized mice resulted in an unaltered arthritis incidence and severity as compared to the control virus AdLuc. Accordingly, no changes in mRNA expression or protein production were observed in CIA mice locally overexpressing IL-22. In contrast, systemic overexpression of IL-22 potently reduced disease incidence and severity, which was also confirmed by histological analysis. Systemic levels of IL-1 $\beta$, IL-17, GM-CSF and MCP1 were unaltered in mice overexpressing IL-22 systemically. However, these mice showed significantly lower serum levels of IFN $\gamma, \mathrm{TNF} \alpha$, MIP1 $\alpha$, and IL-10. Interestingly, the significantly enhanced splenic SOCS3 expression was found to negatively correlate to serum TNF $\alpha$ and MIP1 $\alpha$ levels, which is in line with our hypothesis that that the observed reduction in the cytokine levels is mediated in a SOCS3-dependent manner.

Conclusions With this study, we revealed clear anti-inflammatory effects of IL-22 overexpression during collagen-induced arthritis, which are completely dependent on the systemic route of administration. Additionally, we were the first to show that this protective effect of IL-22 during experimental arthritis is likely orchestrated via up-regulation of the negative regulator SOCS3.

Disclosure of Interest None declared.

\section{P087 THE ANTI-INFLAMMATORY CYTOKINE INTERLEUKIN 37 IS AN ENDOGENOUS INHIBITOR OF TRAINED IMMUNITY}

${ }^{1,2,3} \mathrm{G}$ Cavalli*, ${ }^{4} \mathrm{M}$ Gresnigt, ${ }^{5} \mathrm{~T}$ Nemkov, ${ }^{2} \mathrm{R}$ Arts, ${ }^{5} \mathrm{~A}$ D'Alessandro, ${ }^{6} \mathrm{~S}$ Giugliano, ${ }^{7} \mathrm{E}$ Eisenmensser, ${ }^{1} \mathrm{~L}$ Dagna, ${ }^{2} \mathrm{~L}$ Joosten, ${ }^{2} \mathrm{M}$ Netea, ${ }^{2,3} \mathrm{C}$ Dinarello. ${ }^{1}$ Unit of Immunology, Rheumatology, Allergy and Rare Diseases (UniRAR), Vita-Salute San Raffaele University, Milan, Italy; 'Department of Medicine, Radboud University Medical Center, Nijmegen, Netherlands; ${ }^{3}$ Department of Medicine, University of Colorado Denver, Aurora, CO, USA; ${ }^{4}$ Microbial Pathogenicity Mechanisms, Leibniz Institute for Natural Product Research and Infection Biology, Jena, Germany; ${ }^{5}$ Department of Biochemistry and Molecular Genetics, University of Colorado Denver, Aurora, CO, USA; ${ }^{6}$ Mucosal Immunology and Microbiota Unit, Humanitas University, Milan; 'Department of Biochemistry and Molecular Genetics, University of Colorado Denver, Aurora, CO, Italy

\subsection{6/annrheumdis-2018-EWRR2019.76}

Career situation of first and presenting author Assistant. Introduction Trained immunity (TI) is a de-facto innate immune memory program induced in monocytes/macrophages 